\title{
Stability of a second order consensus algorithm with time delay
}

\author{
Wen Yang, Andrea L. Bertozzi, Xiaofan Wang, Senior member, IEEE
}

\begin{abstract}
In this paper, we investigate a second order consensus problem in a directed graph with non-uniform time delay. This problem arises naturally in real multi-agent systems such as distributed sensing and collective motion. We analyze convergence of the consensus algorithm by frequency domain methods. By using the small $-\mu$ stability theorem, we derive criteria to guarantee convergence. Furthermore, we extend the analysis to higher order systems with time delay. Finally, we present simulations to illustrate theoretical results.

Index Terms- Cooperative control, Consensus problem, time delay, frequency domain.
\end{abstract}

\section{INTRODUCTION}

Consensus means that multiple agents reach an agreement on a common value. In recent years, consensus problems have attracted researchers from diverse areas including physics, control engineering and biology. The role of connectivity stability has been shown to be important for cooperative control consensus algorithms, see e.g., Ren [1-3], Olfati-Saber [4-5], Moreau [6-7] and Cao [8]. Additional work includes cooperative control studies on fully connected graphs [9], rendezvous problem [10-11].

One important challenge is the influence of time delays in the inter-agent information flows. In real systems, time delay always exists due to finite communication speed. Much works has recently been done on consensus algorithms with time-delay, and most of these works have been focused on first order consensus algorithms. For example, a consensus algorithm with uniform constant commensurate delay is analyzed under undirected topology [4]. Ref [12] further extends these results to multiple time-varying delays with switching topologies. In [6], uniform constant non-commensurate delay is analyzed under a directed topology for fixed and switching systems. Ref [13] considers constant non-uniform, non-commensurate delay on a fixed topology.

Consensus algorithms can also be used in cooperative control algorithms where real vehicles often have second order dynamics. However, research results are relatively rare for second/high order consensus problems with time delay. In [14], a second order consensus algorithm with single constant delay is considered for fixed networks. In [15], a

Wen Yang, Xiaofan Wang are with the Department of Automation, Shanghai Jiao Tong University, Shanghai, P R China. Wen Yang also holds a position as visiting student in the Department of Mathematics, University of California, Los Angeles, USA. wenyang@math.ucla.edu, xfwang@sjtu.edu.cn.

Andrea L. Bertozzi is with the Faculty of the Department of Mathematics, University of California, Los Angeles, USA. bertozzi@math.ucla.edu. leader-following consensus problem of a group of second order dynamics agents with time-varying coupling delays is investigated.

In this paper, we consider second order consensus algorithms with time delays. Previous time delay algorithms can be classified as commensurate (e.g. [4]) and non-commensurate (e.g. [6]). Time delay caused by communication constraints can be best modeled by a noncommensurate algorithm, and this is the motivation for our current study. Much of the recent analysis of consensus algorithms with time delay is performed in the time domain. In this paper, we adopt a frequency domain method. The key idea is to transform the time delay system to a feedback interconnection of a linear time-invariant plant and a bounded operator [16-20]. First, we analyze a second order consensus algorithm under a fixed directed network with time-varying delay, and then we investigate the time-invariant delay case. Finally, we extend the problem to higher order systems.

\section{BACKGROUND AND INFORMATION GRAPH}

It is natural to model information exchange among agents of networks by directed/undirected graphs. A relation between the graph Laplacian, a well-known matrix in algebraic graph theory, and special cases of consensus problems are derived in [21-22].

Let $G(v, \varepsilon)$ be a weighted graph of order $N$ with a set of nodes $v=\left\{v_{1}, v_{2}, \ldots, v_{N}\right\}$ and a set of edges $\varepsilon \subset v \times v$. An edge of $G$ is denoted by $e_{i j}=\left(v_{i}, v_{j}\right) \in \mathcal{E}$, which implies that node $i$ can sense node $j$. In this case, node $j$ is the parent node and node $i$ is the child node, but not necessarily vice versa unless the graph is an undirected graph. A directed tree is a directed graph, where every node except one has exactly one parent. The root has no parent, and has a directed path (i.e. a combination of edges in $\varepsilon$ ) to every other node. A directed spanning tree of a directed graph is a directed tree that contains all nodes of the directed graph. A directed graph contains a directed spanning tree if there is a directed spanning tree as a subset of the directed graph, that is, there exists at least one node having directed paths to all of the other nodes.

The nonnegative adjacency matrix $A=\left[a_{i j}\right]$ of a weighted graph specifies the interconnection topology of a network. Let $N_{i}=\left\{v_{j} \in v, a_{i j} \neq 0\right\}$ denote the set of neighbors of node $i$. It is defined by $a_{i i}=0$ and $a_{i j}=\frac{1}{\left|N_{i}\right|}$ if $\left(v_{i}, v_{j}\right) \in \mathcal{E}$ where $i \neq j$. The Laplacian of the weighted graph is defined as 
$L=\left[l_{i j}\right]$, where $l_{i i}=\sum_{j} a_{i j}=1$, and $l_{i j}=-a_{i j}$, where $i \neq j$.

Thus, 1 is always an eigenvalue of $A$ with right eigenvector $[1,1, \ldots 1]^{T} \in R^{N}$, and 1 is a simple eigenvalue of $A$ if and only if $G$ contains a directed spanning tree [21].

Suppose that each agent (node) in the network is a second order integrator with dynamics

$$
\dot{x}_{i}=v_{i}, \quad \dot{v}_{i}=u_{i}, \quad i \in\{1, \ldots . N\}
$$

where $x_{i} \in \mathbb{R}^{n}$ and $v_{i} \in \mathbb{R}^{n}$ are the agent positions and velocities, $u_{i} \in \mathbb{R}^{n}$ is the control input which is taken as

$$
u_{i}=-\sum_{j=1}^{N} a_{i j}\left[\left(x_{i}(t)-x_{j}\left(t-\tau_{i j}(t)\right)\right)+\gamma\left(v_{i}(t)-v_{j}\left(t-\tau_{i j}(t)\right)\right)\right],
$$

where $\gamma>0$ is the damping gain, $\tau_{i j}(t)$ is the non-commensurate non-uniform transmitted information delay from node $j$ to $i$ at time $t$. Here we assume $n=1$ for simplicity. However, all the results hereafter can be derived for $n>1$, using the Kronecker product.

Substituting (2) into (1), the system dynamics is:

$$
\begin{aligned}
{\left[\begin{array}{c}
\dot{X}(t) \\
\dot{V}(t)
\end{array}\right]=\left[\begin{array}{rr}
0_{N \times N} & I_{N} \\
-I_{N} & -\gamma \cdot I_{N}
\end{array}\right] } & {\left[\begin{array}{l}
X(t) \\
V(t)
\end{array}\right] } \\
& +\sum_{k=1}^{q}\left[\begin{array}{ll}
0_{N \times N} & 0_{N \times N} \\
A_{k} & \gamma \cdot A_{k}
\end{array}\right]\left[\begin{array}{l}
X\left(t-\tau_{k}(t)\right) \\
V\left(t-\tau_{k}(t)\right)
\end{array}\right]
\end{aligned}
$$

where $X(t)=\left[x_{1}(t), \cdots, x_{N}(t)\right]^{T}, V(t)=\left[v_{1}(t), \cdots, v_{N}(t)\right]^{T}$, and $\tau_{k}(t) \in\left\{\tau_{i j}(t): i, j \in \underline{N}, i \neq j\right\} \quad$ for $\quad k=1, \ldots ., q \quad$ with $q \leq n(n-1)$, and $A_{k}=\left[A_{k i j}\right]$ is the matrix defined by

$$
A_{k i j}= \begin{cases}a_{i j}, & j \neq i, \tau_{k}(\cdot)=\tau_{i j}(\cdot), \\ 0, & j \neq i, \tau_{k}(\cdot) \neq \tau_{i j}(\cdot), \\ 0, & j=i .\end{cases}
$$

It is easy to see that $\sum_{k=1}^{q} A_{k}=A$. Note that $A$ is a normalized matrix, i.e., $\sum_{j=1}^{N} a_{i j}=1, \forall i \in \underline{N}, \underline{N}=\{1,2, \ldots, N\}$.

Remark 1: Here $q=|\varepsilon|(|\cdot|$ is the cardinality of $\varepsilon)$ is the total number of different time delays. If the network is a directed complete graph, and all the time-delays are different, then $q$ attains its maximum, i.e., $N(N-1)$.

Furthermore, we denote $U(t)=[X(t), V(t)]^{T}$,

$$
\Gamma=\left[\begin{array}{cc}
0_{N \times N} & I_{N} \\
-I_{N} & -\gamma \cdot I_{N}
\end{array}\right], \mathrm{H}_{k}=\left[\begin{array}{cc}
0_{N \times N} & 0_{N \times N} \\
A_{k} & \gamma \cdot A_{k}
\end{array}\right],
$$

then (3) can be rewritten as

$$
\dot{U}(t)=\Gamma \cdot U(t)+\sum_{k=1}^{q} \mathrm{H}_{k} \cdot U\left(t-\tau_{k}(t)\right)
$$

where $0 \leq \tau_{k}(t) \leq \tau_{0}, \dot{\tau}_{k}(t) \leq d_{k}, k \in \underline{q}, \underline{q}=\{1,2, \ldots, q\}$.

\section{MAIN RESULTS}

\section{A. Time-varying delay case}

In this section, we consider a second order consensus algorithm with non-uniform time delay under a fixed topology. Stability analysis of time-delay systems is a large research topic, and there have been fruitful results derived by different approaches including the Lyapunov-type approach [23-26] in the time domain and the small gain theorem and structured singular value in the frequency domain [16-20]. In the following, we analyze the consensus problem (4) with time-varying delay based on the small $-\mu$ stability theorem.

One difficulty for time-varying delay systems is that the delay operator is no longer energy-preserving (i.e., not bounded) and the gain of delay operator might be infinite, thus we can not directly use the small $-\mu$ stability theorem. The essential idea here is to transform the delay system to a linear time-invariant plant and an above bounded time delay operator, and then a small $-\mu$ approach for proving stability of systems can be applied.

Let $L_{2}^{l}[0, \infty)$ be the space of $R^{l}$-valued functions $f:[0, \infty) \rightarrow R^{l}$ of finite energy

$$
\|f\|^{2}=\int_{0}^{\infty}|f(t)|^{2} d t<\infty .
$$

$L_{2 e}^{l}[0, \infty)$ is the extension of the space $L_{2}^{l}[0, \infty)$, its members only need to be square integrable on finite intervals. The gain of an operator (function) $F: L_{2 e}^{a}[0, \infty) \rightarrow L_{2 e}^{b}[0, \infty)$ is

$$
\|F\|=\sup \left\{\frac{\|F(f)\|}{\|f\|}: f \in L_{2 e}^{a}[0, \infty), f \neq 0\right\} .
$$

Consider the time-varying delay system (4), by taking a simple modification and Laplace transformation, we can get a feedback interconnection of a linear time-invariant plant with a delay operator as follows:

$$
\tilde{U}=G(s) \Delta(\tilde{U})
$$

where $\tilde{U}=\left[\tilde{U}_{1}^{T}, \tilde{U}_{2}^{T}, \ldots, \tilde{U}_{q}^{T}\right]^{T}, \tilde{U}_{1} \in \mathbb{R}^{2 N}, G(s)$ is the transfer function for multiple input multiple output system ,

$$
G(s):=[I, \ldots, I]^{T}(s I-\Gamma-H)^{-1}\left[H_{1}, \ldots, H_{q}\right],
$$

with $\sum_{k=1}^{q} H_{k}=H$. We denote the time delay operator $\vartheta_{\tau i, i \in \underline{q}}(x):=x(t)-x\left(t-\tau_{i}(t)\right) ; \Delta=\operatorname{diag}\left\{\vartheta_{\tau 1}, \vartheta_{\tau 2}, \cdots \vartheta_{\tau q}\right\}$ where $\vartheta_{\tau i}$ are causal operators on $L_{2 e}^{l}[0, \infty)$ with bounded gain.

Lemma 1([19]): Consider the set of differentiable functions $\mathfrak{\aleph}:=\left\{s(t)\left|s(t) \in\left[0, \tau_{0}\right],\right| \dot{s}(t) \leq d \mid, \forall t\right\}$. Then the following equality holds: $\sup _{\tau(t) \in \mathbb{\aleph}}\left\|\vartheta_{\tau} \circ \frac{1}{s}\right\|_{L_{2}}=\tau_{0}$.

In the following discussion, $\omega$ is the frequency of the signal after Fourier transform in the time domain.

Theorem 1: Consider the time-varying delay system (4). Suppose that the network $G$ contains a spanning tree, and 
i)

$$
\tau_{0}<\frac{1}{\omega \cdot \max _{i \in \underline{N}} \sum_{k=1}^{p_{i}}\left|\left(j \omega-\frac{\gamma \mu_{i} \pm \sqrt{\gamma^{2} \mu_{i}^{2}+4 \mu_{i}}}{2}\right)^{-k}\right| \cdot(1+\gamma)}
$$

$\forall \omega \in(0,+\infty)$

ii) $\gamma>\max _{\mu_{i} \neq 0} \sqrt{\frac{2}{\left|\mu_{i} \cdot \cos \left(\frac{\pi}{2}-\tan ^{-1} \frac{\operatorname{Re}\left(\mu_{i}\right)}{\operatorname{Im}\left(\mu_{i}\right)}\right)\right|}}$.

where $\tau_{0}$ is the upper bound for time-varying delay, and $\mu_{i}$ are the eigenvalues of $-L$.Then the system achieves consensus.

Proof: Firstly, we investigate the stability of $\Gamma+H$ since the system must be stable for $\tau_{i}(t)=0$.Note that

$\Gamma+H=\left[\begin{array}{cc}0 & I_{N} \\ -L & -\gamma \cdot L\end{array}\right]_{2 N \times 2 N}, H=\sum_{k=1}^{q} H_{k}=\left[\begin{array}{cc}0_{N \times N} & 0_{N \times N} \\ A & \gamma \cdot A\end{array}\right]$.

It is easy to see that $\Gamma+H$ has two zero eigenvalues iff $-L$ has a simple zero eigenvalue. This occurs when the network topology contains a spanning tree [2]. Moreover, if condition ii) holds [2], all the other eigenvalues of $\Gamma+H$ have negative real parts. Then the system with zero delay is marginally stable, which implies some sustained constant persists at zero frequency. Furthermore, we investigate whether all the signals at non-zero frequency (i.e. $\omega \in(0,+\infty))$ will die out.

Let $\Lambda$ be the Jordan form of $\Gamma+H, \mu_{i}$ be the eigenvalues of $-L$ and $1+\mu_{i}$ be the eigenvalues of $A, i \in \underline{N}$. By the definition of the Laplacian matrix, we know that $\operatorname{Re} \mu_{i} \leq 0$, thus

$$
\begin{aligned}
\|G(s) \Delta\| & \leq\left\|[I, \ldots, I]^{T}(s I-\Lambda)^{-1}\left[s H_{1}, \ldots, s H_{q}\right] \Delta \circ \frac{1}{s}\right\| \\
& \leq\left\|[I, \ldots, I]^{T}(s I-\Lambda)^{-1}\left[s H_{1} \vartheta_{\tau 1} \circ \frac{1}{s}, \ldots ., s H_{q} \vartheta_{\tau q} \circ \frac{1}{s}\right]\right\| \\
& \leq \sup _{\omega}\left\{\max \left\|(s I-\Lambda)^{-1} s H\right\| \cdot \max _{i \in \underline{q}}\left\|\vartheta_{\tau i} \circ \frac{1}{s}\right\|\right\} .
\end{aligned}
$$

According to the definition of the adjacency matrix, $\|H\|_{\infty}=1+\gamma$. Furthermore, the eigenvalues of $\Gamma+H$ are given by $\lambda_{i \pm}=\frac{\gamma \mu_{i} \pm \sqrt{\gamma^{2} \mu_{i}^{2}+4 \mu_{i}}}{2}, i \in \underline{N}, \underline{N}=\{1,2, \ldots, N\}$. Also note that $\|s I-\Gamma-H\|_{\infty}^{-1}=\max _{i \in 2 \underline{N}} \sum_{k=1}^{p_{i}}\left|\left(s-\lambda_{i}\right)^{-k}\right|$, where $p_{i}$ is the algebraic multiplicity of $\lambda_{i}$. Hence it follows from the small- $\mu$ stability theorem and lemma 1, $\max _{i} \sum_{k=1}^{p_{i}}\left|\left(j \omega-\frac{\gamma \mu_{i} \pm \sqrt{\gamma^{2} \mu_{i}^{2}+4 \mu_{i}}}{2}\right)^{-k}\right| \cdot(1+\gamma)<\frac{1}{\tau_{0} \omega} \quad$ should be guranteed. Therefore, the system is stable for $\tau_{i}(t) \in\left[0, \tau_{0}\right]$ if condition i) holds for $\forall \omega \in[0,+\infty)$. The above condition can be guaranteed if there exists an appropriate $\tau_{0}$ for any $\omega \in(0,+\infty)$, then the non-zero frequency will die out. This is not necessarily guaranteed at $\omega=0$. Thus by taking Laplace transforms of both sides, system (1) and (2) can be expressed in the s domain as

$$
s^{2} X(s)=-X(s)-\gamma \cdot s X(s)+A \cdot X(s)+\gamma \cdot A \cdot s \cdot X(s) .
$$

Then we can get

$$
-L \cdot \bar{X}=0 \text { for } s=j \omega=0
$$

where $-L=-I_{N}+A$, and $\bar{X}$ is the DC-offset, thus $\bar{X}$ is an eigenvector of $-L$ associated with eigenvalue zero. Since the network topology contains a spanning tree, $-L$ has exactly one zero eigenvalue, which implies $-L$ has only one linearly independent eigenvector $\quad \bar{X} \in \operatorname{span}\left\{[1, \ldots .1]^{T}\right\} \quad$ and $\bar{x}_{1}=\ldots=\bar{x}_{N}=c$, where $c \in \mathbb{R}$ is an unknown constant. Furthermore, it is readily seen that $\bar{v}_{1}=\bar{v}_{2}=\ldots=\bar{v}_{N}=0$. Hence the small- $\mu$ stability theorem proves Theorem 1 .

Remark 2: If all $\lambda_{i}$ are real with $\gamma^{2} \mu_{i}^{2}+4 \mu \geq 0$, then we can get $\tau_{0}<1 /(\gamma+1)(\omega+1)$. In the case that some eigenvalues $\mu_{i}$ are complex and condition ii) holds, we order all the eigenvalues as $\mu_{1}=0>\operatorname{Re} \mu_{2} \geq \cdots \geq \operatorname{Re} \mu_{N} \geq-2$, where $-\mu_{2}$ is the algebraic connectivity of the network [4], then condition i) becomes

$$
\sum_{k=1}^{p_{2}}\left|\left(j \omega-\frac{\gamma \mu_{2} \pm \sqrt{\gamma^{2} \mu_{2}^{2}+4 \mu_{2}}}{2}\right)^{-k}\right| \cdot(\gamma+1)<\frac{1}{\tau_{0} \omega} .
$$

The bigger $-\mu_{2}$ is, the more connected the network is. It is easy to see that the delay bound $\tau_{0}$ increases as $-\mu_{2}$ increases, which implies the network with higher connectivity is more robust to time delay.

\section{B. Time-invariant delay case}

In the following, we derive a less conservative delay-dependent condition for time invariant delay system by a similar method to Theorem 1. Here, we consider system (4) with $\tau_{i j}(t)$ constant, and suppose that $\tau_{i} \in[0, h]$ with $h$ is the delay maximum. To proceed with our analysis, we need the following preliminary results (refer to [16]).

Definition 1: The structured singular value of $M$ with respect to $\chi_{q}(\gamma)$, denoted by $\mu \chi_{q}(M)$, is defined as

$\mu \chi_{q}(M):= \begin{cases}0 & \text { if } \operatorname{det}(I-M \Delta) \neq 0 \forall \Delta \in \chi_{q}(\infty) \\ \min _{\Delta \in \chi_{q}(\infty)}\{\bar{\sigma}(\Delta): \operatorname{det}(I-M \Delta)=0\}^{-1} & \text { otherwise }\end{cases}$ where $\bar{\sigma}(\Delta)$ denotes the largest singular value of $\Delta$.

Definition 2: $\chi_{q}(\gamma):=\left\{\operatorname{diag}\left(\delta_{1} I_{k_{1}}, \ldots, \delta_{k} I_{k_{q}}\right): \delta_{k} \in C,\left|\delta_{k}\right| \leq \gamma\right\}$ denotes the family of block diagonal $m \times m$ matrices, satisfying $\sum_{i=1}^{q} k_{i}=m$.

Lemma 2: $\mu \chi_{q}(M)=\max _{Q \in \mathbb{Q}} \rho(M Q)$, where

$$
\mathbb{Q}:=\left\{\operatorname{diag}\left(\delta_{1} I_{k_{1}}, \ldots . ., \delta_{k} I_{k_{q}}\right): \delta_{k} \in C,\left|\delta_{k}\right|=1\right\} .
$$


The following theorem follows immediately by applying the small- $\mu$ theorem for systems with frequency-dependent uncertainty bounds (see, [18, Theorem 3]) to the time invariant delay system.

Theorem 2: Consider the time-invariant delay system (4) with $\tau_{i j}$ constant. Suppose that

i) the network $G$ contains a spanning tree,

ii) $\max _{i \in \underline{N}}\left|\left(j \omega-\frac{\gamma \mu_{i} \pm \sqrt{\gamma^{2} \mu_{i}^{2}+4 \mu_{i}}}{2}\right)^{-1}\right| \cdot \gamma \cdot\left(1+\mu_{i}\right) \cdot \sin \frac{h \omega}{2}<\frac{1}{2}$,

$\forall 0 \leq \omega \leq \pi / h$,

iii) $\max _{i \in \underline{N}}\left|\left(j \omega-\frac{\gamma \mu_{i} \pm \sqrt{\gamma^{2} \mu_{i}^{2}+4 \mu_{i}}}{2}\right)^{-1}\right| \cdot \gamma \cdot\left(1+\mu_{i}\right)<\frac{1}{2}$,

$\forall \omega \geq \pi / h$,

iv) $\gamma>\max _{\mu_{i} \neq 0, i \in \underline{N}} \sqrt{\frac{2}{\left|\mu_{i} \cdot \cos \left(\frac{\pi}{2}-\tan ^{-1} \frac{\operatorname{Re}\left(\mu_{i}\right)}{\operatorname{Im}\left(\mu_{i}\right)}\right)\right|}}$.

Then the system achieves consensus.

Proof: According to [18], we can get the system transfer matrix as

$$
P(s)=C\left(s I-\Gamma-\sum_{k=1}^{q} H_{k}\right)^{-1} B,
$$

where $B=\left[\begin{array}{llll}B_{1} & B_{2} & \cdots & B_{q}\end{array}\right], C=\left[\begin{array}{llll}C_{1}^{T} & C_{2}^{T} & \cdots & C_{q}^{T}\end{array}\right]^{T}$, and $H_{i}=B_{i} C_{i}, B_{i} \in \mathbb{R}^{2 N \times r_{i}}, C_{i} \in \mathbb{R}^{r_{i} \times 2 N}, r_{i}=\operatorname{rank}\left(H_{i}\right)$. Here, we use a trivial factorization: $B_{i}=H_{i}$ and $C_{i}=I$. Moreover, $L(\omega)=\operatorname{diag}\left\{l_{1}(\omega) I_{r_{1}}, l_{2}(\omega) I_{r_{2}}, \cdots, l_{q}(\omega) I_{r_{q}}\right\}, j \in \underline{q}$, $l_{j}(\omega)=2, \forall \omega \geq \pi / h$ and $l_{j}(\omega)=2 \sin \frac{h \omega}{2}, \forall 0 \leq \omega \leq \pi / h$. Again, it is shown in [18] that the delay system is stable for all $\tau_{i} \in[0, h]$ if $\dot{U}(t)=\Gamma \cdot U(t)+\sum_{k=1}^{q} \mathrm{H}_{k} \cdot U(t)$ is stable and $\mu \chi_{q}(L(\omega) P(j \omega))<1, \forall \omega, i \in \underline{q}$. It follows from Theorem 1 that the matrix $\Gamma+H$ is marginally stable if condition iv) is satisfied.

Note that

$$
\rho(L(\omega) P(j \omega) Q) \leq \max _{i \in \underline{N}, j \in \underline{q}}\left|j \omega I-\lambda_{i_{ \pm}}\right|^{-1} \cdot \gamma \cdot\left(1+\mu_{i}\right) \cdot l_{j}(\omega)
$$
according to Lemma 2 , we can get

$$
\begin{aligned}
\mu \chi_{q}(L(\omega) P(j \omega)) & =\max _{Q \in \mathbb{Q}} \rho(L(\omega) P(j \omega) Q) \\
& \leq \max _{i \in \underline{N}, j \in \underline{q}}\left|j \omega I-\lambda_{i_{ \pm}}\right|^{-1} \cdot \gamma \cdot\left(1+\mu_{i}\right) \cdot l_{j}(\omega),
\end{aligned}
$$

where $\lambda_{i}$ is the eigenvalue of $\Gamma+\mathrm{H}$. The analysis for zero frequency $(\omega=0)$ is as in Theorem 1. Hence the time invariant delay system can reach consensus if $\max _{i \in \underline{N}, j \in q}\left|j \omega I-\lambda_{i_{ \pm}}\right|^{-1} \cdot \gamma \cdot\left(1+\mu_{i}\right) \cdot l_{j}(\omega)<1, \forall \omega \in(0,+\infty)$.

We can also derive a delay-independent stability result for the fixed delay problem. For example, according to Lemma 3 in [16], we can prove the following Theorem.
Theorem 3: Assume that the fixed network topology of $G$ contains a spanning tree, then the time-invariant delay system (4) with $\tau_{i j}$ constant can achieve consensus asymptotically if $\gamma>2$ for all $\omega \in(0,+\infty)$ and $\gamma<\sqrt{\left(\omega^{4}-2 \omega^{2}+1\right) /\left(\omega^{2}-2\right)}$ for all $\omega \in(\sqrt{2},+\infty)$.

\section{HIGH ORDER CONSENSUS PROBLEM WITH TIME DELAYS}

In this section, we extend the second order results to higher order consensus problems with time delays. One motivation for studying higher order consensus problems comes from flocking and swarming. For example, in a group of birds, each individual bird often suddenly accelerates or decelerates, thus the group needs to achieve not only velocity consensus but also acceleration consensus. Ren et.al. propose consensus algorithms for high order system, and derive sufficient and necessary conditions for consensus [3]. Here, we study the high order consensus problem with time delays.

Consider each agent of a graph to be a dynamic high order integrator, i.e.,

$$
\begin{aligned}
& \dot{x}_{i}^{(0)}=x_{i}^{(1)}, \\
& \vdots \\
& \dot{x}_{i}^{(l-2)}= x_{i}^{(l-1)}, \\
& \dot{x}_{i}^{(l-1)}=u_{i}, \quad i \in\{1, \ldots . ., N\},
\end{aligned}
$$

where $x_{i}^{(k)} \in \mathbb{R}^{n}, k=0,1, \ldots, l-1$, is the state of each agent, $u_{i} \in \mathbb{R}^{n}$ is the control input and has the following form:

$$
u_{i}=-\sum_{j=1}^{N} a_{i j}\left[\sum_{k=0}^{l-1} \gamma_{k}\left(x_{i}^{(k)}-x_{j}^{(k)}\left(t-\tau_{i j}(t)\right)\right)\right], \quad i \in\{1, \ldots, N\}
$$

where $a_{i j}$ and $\tau_{i j}(t)$ are as in section III, $\gamma_{k}>0$ is a constant damping gain. Here, we also assume $n=1$ for simplicity. Substituting (7) into (6) and stacking up all the individual agents' dynamics, we write the whole system dynamics as:

$$
\dot{U}(t)=\Gamma \cdot U(t)+\sum_{k=1}^{q} \mathrm{H}_{k} \cdot U\left(t-\tau_{k}(t)\right),
$$

where $U(t)=\left[\left(\dot{x}^{(0)}\right)^{T},\left(\dot{x}^{(1)}\right)^{T}, \ldots,\left(\dot{x}^{(l-1)}\right)^{T}\right]^{T}, \tau_{k}(t)$ and $A_{k}$ are shown in section III, and

$$
\begin{gathered}
\Gamma=\left[\begin{array}{ccccc}
0_{N} & I_{N} & 0_{N} & \cdots \cdots & 0_{N} \\
0_{N} & 0_{N} & I_{N} & \cdots \cdots & 0_{N} \\
\vdots & \vdots & \vdots & & \vdots \\
0_{N} & 0_{N} & 0_{N} & \cdots \cdots & I_{N} \\
-\gamma_{0} I_{N} & -\gamma_{1} I_{N} & -\gamma_{2} I_{N} & \cdots \cdots & \gamma_{l-1} I_{N}
\end{array}\right]_{I N \times I N} \\
H_{k}=\left[\begin{array}{ccccc}
0_{N} & 0_{N} & 0_{N} & \cdots \cdots & 0_{N} \\
0_{N} & 0_{N} & 0_{N} & \cdots \cdots & 0_{N} \\
\vdots & \vdots & \vdots & & \vdots \\
0_{N} & 0_{N} & 0_{N} & \cdots \cdots & 0_{N} \\
\gamma_{0} A_{k} & \gamma_{1} A_{k} & \gamma_{2} A_{k} & \cdots \cdots & \gamma_{l-1} A_{k}
\end{array}\right]_{I N \times I N}
\end{gathered}
$$

Theorem 4: Consider the time-varying delay system (8). Suppose that 
i) the network $G$ contains a spanning tree,

ii) $\tau_{0}<\frac{1}{\max _{j \in \underline{I} \underline{\underline{N}}} \sum_{k=1}^{p_{j}}\left|\left(j \omega-\lambda_{j}\right)^{-k}\right| \cdot \omega \cdot \sum_{i=1}^{l-1} \gamma_{i}}$, for $\forall \omega \in(0,+\infty)$,

iii) $\Gamma+H$ has only $l$ zero eigenvalues and all the other $l(N-1)$ eigenvalues have negative real parts.

Then the system achieves consensus. Here $\lambda_{i}$ are eigenvalues of $\Gamma+H$.

The following Corollary follows from Theorem 2 by applying the small- $\mu$ theorem for a time-invariant delay system.

Corollary 5: Consider the time-invariant delay system. Suppose that

i) the network $G$ contains a spanning tree,

ii) $\max _{i}\left|j \omega I-\lambda_{i}\right|^{-1} \cdot \sin \frac{h \omega}{2}<\frac{1}{2}, \forall 0 \leq \omega \leq \pi / h$,

iii) $\max _{i}\left|j \omega I-\lambda_{i}\right|^{-1}<\frac{1}{2}, \forall \omega \geq \pi / h \quad i \in \underline{N}$,

iv) $\Gamma+H$ has only $l$ zeros and all the other $l(N-1)$ eigenvalues have negative real parts.

Then the system achieves consensus.

Also, we extend the delay-dependent criteria to high-order case. Note that $\lambda_{j}$ and $\mu_{i}$ are eigenvalues of $\Gamma+H$ and $-L$, respectively.

Theorem 6: Assume that the network topology of $G$ contains a spanning tree, then the system (8) with time invariant delays (i.e. $\tau_{k}(t)$ is constant) can achieve consensus asymptotically if $\max _{j}\left|\left(j \omega-\lambda_{j}\right)^{-1}\right| \cdot \max _{i} \gamma_{l-1} \cdot\left|\left(1+\mu_{i}\right)\right|<1, j \in l \underline{N}, i \in \underline{N}$ holds for $\forall \omega \in(0,+\infty)$.

\section{EXAMPLES AND SIMULATION RESULTS}

In this section, we illustrate the results derived in the above sections by numerical simulations using the Simulink toolbox in Matlab. Here, we consider three network topologies as shown in Fig.1 with each network containing a spanning tree. Example 1. (Time-invariant delay case) Each node is a second order dynamic integrator as (3). By Theorem 3, if $\gamma$ is bounded, all the position states will reach the same constant, and all the velocity states will reach zero, i.e., the system will rendezvous (a specific consensus problem [10-11]).

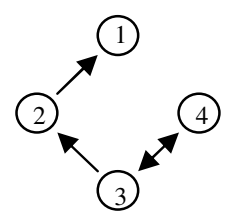

(a)

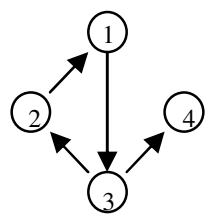

(b)

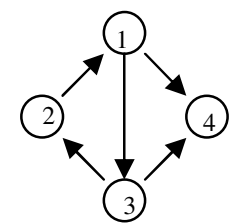

(c)
Fig. 1. Three examples of directed graphs contain spanning tree: the first graph has $v_{3}, v_{4}$ as root nodes, the second and third graphs have $v_{1}, v_{2}, v_{3}$ as root nodes.

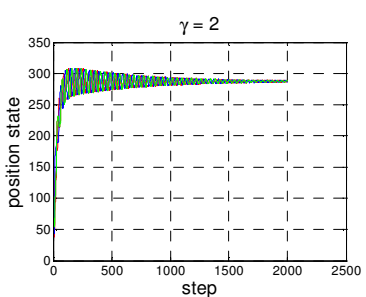

(a)

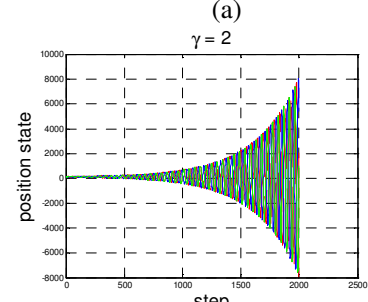

(c)

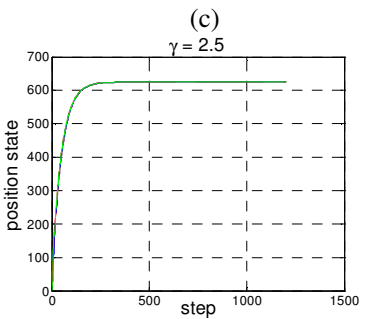

(e)

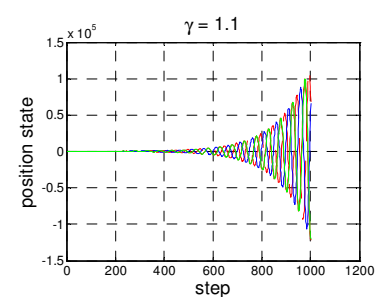

(b)

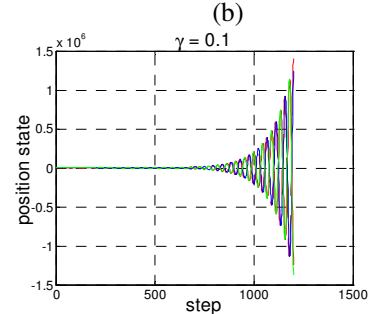

(d)

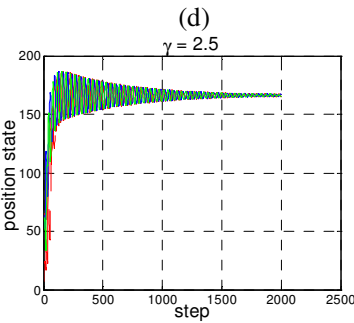

(f)
Fig. 2. Position trajectories of agents in Fig.1. Here, (a)-(c) are with topology b. Simulation (a) and (b) have the same time delays $\tau_{12}=1, \tau_{23}=0.9, \tau_{31}=0.8, \tau_{43}=1$, and (c) has time delays $\tau_{12}=1.6, \tau_{23}=1.5, \tau_{31}=1.3, \tau_{43}=1.2$. Simulation (d)-(f) are with topology a, and (d), (e) with time delays $\tau_{12}=0.1, \tau_{23}=0.4, \tau_{34}=0.6$, $\tau_{43}=0.2$, while (f) has time delay $\tau_{12}=0.9, \tau_{23}=1, \tau_{34}=0.8, \tau_{43}=1$.

In the simulations, the time step size is 0.1 seconds. According to Theorem 2, we estimate the lower bound for the gain $\gamma$ and the maximal delay $h$ for topology (b) in Fig. 1 to be $\gamma \geq 1.1547$ and $h<1.57$. As shown in Fig. 2 (a)-(c), the system can not achieve consensus when $\gamma$ is less than the estimation or the time delay is above $h$. As Fig. 2 (d) and (f) show, the system will always achieve position consensus if $2<\gamma<4$ but fails in (c) with small $\gamma$. However, it should be noted that the condition in Theorem 3 is very conservative since it is delay-independent, which implies that position consensus might also be achieved for some $\gamma$ outside the limited range.

Example 2. (Time-varying delay case)

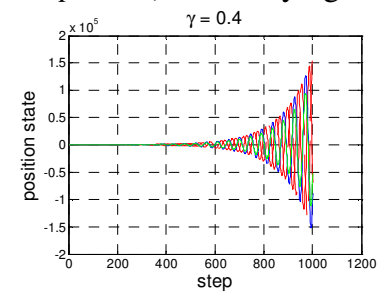

(a)

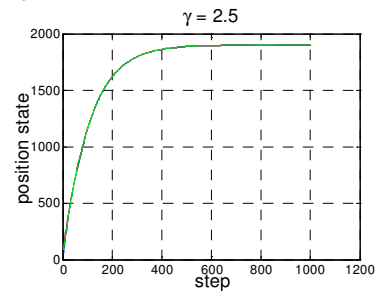

(b) 


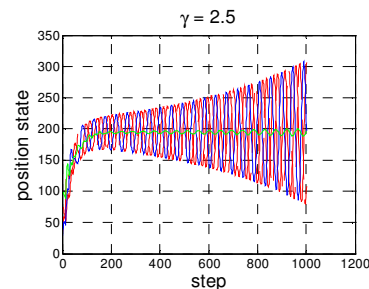

(c)

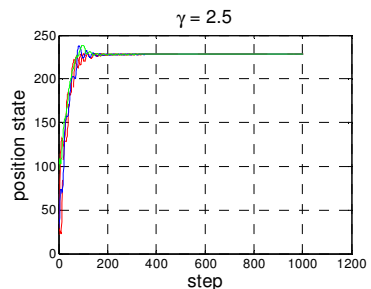

(d)
Fig. 3. Position trajectories of agents with topology (b) and (c) in Fig. 1. Here, (a), (b), (d) has maximal time delay $\tau_{0}=0.2$. (c) has maximal time delay $\tau_{0}=1$. And (a)-(c) are with topology b, (d) is with topology c.

According to Theorem 1, the system achieves consensus if condition i) and ii) hold. Fig. 3 (a) shows that the system cannot rendezvous when $\gamma$ is too small since condition ii) is not satisfied. With the same $\gamma$, (b) can reach rendezvous while (c) with large $\tau_{0}$ fails. It is easy to see that the convergence time in (b) is longer than the one in (d), the heuristic reason is that topology $\mathrm{c}$ is more connected than topology $\mathrm{b}$, which implies the more connected the network is, the more robust it is to time-varying delay.

\section{CONCLUSIONS}

In this paper, we investigate a second order consensus problem with non-uniform time delays and directed topology. Under the assumption that the network has a spanning tree, we provide a bound for the time-delay and the velocity coupling gain to guarantee consensus. We further extend the results to higher order consensus problems with time delays. It should be noted that the bounds for delay are conservative and the final equilibrium for the time-delay case is unknown. In the context of the algorithms considered here, the velocity equilibrium is zero. However, in some cooperative control algorithms, it is desirable to have non-zero velocity (see e.g. [8]). This problem as well as stability of nonlinear consensus algorithms are additional topics for future research.

\section{ACKNOWLEDGMENT}

This research is supported by ARO MURI grant 50363-MA-MURI and ONR grant N000140610059.

\section{REFERENCES}

[1] W. Ren and R. W. Beard, "Consensus seeking in multiagent systems under dynamically changing interaction topologies," IEEE Trans. on Automatic Control, vol. 50, no. 5, pp. 655-661, May 2005

[2] W. Ren and E. M. Atkins, "Distributed multi-vehicle coordinated control via local information exchange," International Journal of Robust and Nonlinear Control, vol. 17, no.10, pp. 1002-1033, July 2007.

[3] Wei Ren, Kevin L. Moore, and YangQuan Chen, "High-Order and Model Reference Consensus Algorithms in Cooperative Control of Multi-Vehicle Systems", ASME Journal of Dynamic Systems, Measurement, and Control, vol. 129, no. 5, pp. 678-688, September 2007.

[4] R. Olfati-Saber and R. M. Murray. "Consensus Problems in Networks of Agents with Switching Topology and Time-Delays," IEEE Trans. on Automatic Control, vol. 49, no. 9, pp. 1520-1533, September 2004
[5] R. Olfati-Saber, "Flocking for multi-agent dynamic systems: Algorithms and theory," IEEE Transactions on Automatic Control, vol. 51, no. 3, pp. 401-420, March 2006.

[6] L. Moreau, "Stability of continuous-time distributed consensus algorithms." In Proc.of the 43nd IEEE Conference on Decision and Control, Atlantis, Paradise Island, Bahamas, 2004, pp. 3998-4003.

[7] L. Moreau, "Stability of multiagent systems with time-dependent communication links," IEEE Trans. on Automatic Control, vol. 50, no. 2, pp. 169-182, February 2005.

[8] M. Cao, A. S. Morse, and B. D. O. Anderson, "Reaching a consensus using delayed information." In Proc.of the 45nd IEEE Conference on Decision and Control, San Diego, 2006, pp. 3375-3380.

[9] Cucker. F, Smale. S, "Emergent behavior in Flocks," IEEE Trans. On Automatic Control, vol. 52, no. 5, pp. 852-862, May 2007.

[10] J. Lin, A. S. Morse, and B. D. O. Anderson, "The multi-agent rendezvous problem -part 1: The synchronous case," SIAM J. on Control and Optimization, vol. 46, no.6, pp. 2096-2119, December 2007

[11] J. Lin, A. S. Morse, and B. D. O. Anderson, "The multi-agent rendezvous problem -part 2: The asynchronous case," SIAM J. on Control and Optimization, vol. 46, no.6, pp. 2120-2147, December 2007.

[12] Yuan Gong Sun, Long Wang and Guangming Xie, "Average consensus in networks of dynamic agents with switching topologies and multiple time-varying delays," Systems and Control Letters, vol. 57, no. 2, pp.175-183, February 2008.

[13] Dongjun Lee and Mark W. Spong, "Agreement with Non-Uniform Information Delays", In Proc. of the 2006 American Control Conference, Minnesota, USA, June 14-16, 2006.

[14] Feng Xiao and Long Wang, "Dynamic behavior of discrete-time multiagent systems with general communication structures", Physica A, vol. 370, no. 2, pp. 364-380, October 2006.

[15] Jiangping Hu and Yiguang Hong, "Leader-following coordination of multi-agent systems with coupling time delays," Physica A, vol. 374, no. 2, pp. 853-863, 2007.

[16] Jie Chen and Haniph A. Latchman, "Frequency Sweeping Tests for Stability Independent of Delay," IEEE Trans. on Automatic Control, vol. 40, no. 9, pp. 1640-1645, September, 1995.

[17] Alexandre Megretski and Anders Rantzer, "System Analysis via Integral Quadratic Constraints," IEEE Trans. on Automatic Control, vol. 42, no. 6, pp. 819-830, June 1997.

[18] Yun-Ping Huang and Kemin Zhou, "Robust Stability of Uncertain Time-delay Systems," IEEE Trans. on Automatic Control, vol. 45, no. 11, pp. 2169-2173, November 2000

[19] Chung-Yao Kao and Anders Rantzer, "Stability analysis of systems with uncertain time-varying delays," Automatica, vol. 43, no.6, pp. 959-970, June 2007.

[20] Chung-Yao Kao and Bo Lincoln, "Simple stability criteria for systems with time-varying delays," Automatica, vol. 40, no.8, pp. 1429-1434, August 2004.

[21] R. Merris, "Laplacian matrices of graphs: A survey," Linear Alg. Applicant., vol. 197, no.11, pp. 143-176, 1994.

[22] R. Horn and C. Johnson, Matrix Analysis. Cambridge, U.K.: Cambridge Univ. Press, 1985.

[23] Jean-Pierre Richard, "Time-delay systems: an overview of some recent advances and open problems", Automatica, vol. 39, no.10, pp. 1667-1694, October 2003.

[24] Fridman, E., and Shaked, U., "Delay-dependent stability and $H_{\infty}$ control: Constant and time-varying delays," International Journal of Control, vol. 76. no. 1, pp. 48-60, 2003.

[25] Gu, K., and Han, Q.-L., "Discretized Lyapunov functional for linear uncertain systems with time-varying delay", In Proc. of the American Control Conference, 2000, pp. 1375-1379.

[26] Kim, J.-H., "Delay and its time-derivative dependent robust stability of time-delayed linear systems with uncertainty," IEEE Trans. on Automatic Control, vol. 46, no. 5, pp. 789-792, May 2001. 